\title{
Immunohistochemical features and staging of early gastric cancer
}

\author{
Simona Gurzu ${ }^{1}$, Janina Orlowska ${ }^{2}$, Haruhiko Sugimura ${ }^{3}$, Tivadar Bara ${ }^{1}$, Zoltan Szentirmay ${ }^{4}$, \\ Wladyslaw Januszewicz ${ }^{2}$, Tivadar Jr. Bara ${ }^{1}$, Janos Szederjesi ${ }^{1}$, Ioan Jung ${ }^{1}$
}

\author{
${ }^{1}$ University of Medicine and Pharmacy, Tirgu-Mureş, Romania \\ ${ }^{2}$ Maria Sklodowska-Curie Memorial Cancer Centre and Institute of Oncology, \\ Warsaw, Poland \\ ${ }^{3}$ Hamamatsu University School of Medicine, Hamamatsu, Japan \\ ${ }^{4}$ National Institute of Oncology, Budapest, Hungary
}

Submitted: 28 November 2015

Accepted: 5 February 2016

Arch Med Sci 2017; 13, 6: 1373-1382

DOI: 10.5114/aoms.2016.58665

Copyright $\odot 2016$ Termedia \& Banach

\section{Abstract}

Introduction: The aim of this study was to analyze the particularities of early gastric cancer (EGC) and their importance for staging, prognosis, and therapy.

Material and methods: A total of $338 \mathrm{GCs}$ diagnosed and surgically removed in three medical institutes from Eastern Europe were retrospectively examined, and the EGCs were further examined. Besides the demographic factors and tumor characteristics, immunostains were performed with E-cadherin, HER-2, p53, Ki67, MLH-1, MSH-2, COX-2, VEGF-A, CD31, and CD105.

Results: From the $338 \mathrm{GCs}, 29$ were EGCs, the average being similar in Poland and Hungary (12.37\% and $13.33 \%$ respectively) but lower in Romania (5.61\%). The rate of lymph node metastases was $20.69 \%(n=6)$. Two of the cases presented liver metastases, both of them having a multifocal aspect. In 1 of these cases, limited to the mucosa, intramural carcinomatosis of the lymph vessels was seen in submucosa, muscularis propria, and subserosa. COX-2 positivity was observed in 14 (48.72\%) cases. COX-2 was directly correlated with microvessel density determined with CD31 $(p<0.001)$ and CD105 $(p=0.03)$. Same correlation with CD31 and CD105 was seen for HER-2 ( $p=0.03$ and $p=0.0007)$. The only negative independent prognostic factors for overall survival were tumor localization at the proximal stomach and male gender, regardless of age.

Conclusions: In EGCs, intramural carcinomatosis of the lymph vessels and multifocality should be separately described in every surgical pathology report, as indicators of aggressiveness. Microsatellite status, E-cadherin, HER-2, p53, and Ki67 do not have prognostic value in EGC, but the highly angiogenic pattern is a possible therapeutic target.

Key words: early gastric cancer, carcinomatosis, angiogenesis, staging, carcinomatous lymphangitis.

\section{Introduction}

Despite the gradual development of gastric cancer (GC) screening programs, most of the patients with GC are still diagnosed in advanced stages, with a 5-year survival rate of only $10 \%$ [1].

The name "early gastric cancer" (EGC) suggests a localized tumor with a potential for complete cure after complete resection, i.e., a low risk of lymph node metastases [2, 3]. For EGCs, endoscopic mucosal resec-

\author{
Corresponding author: \\ Prof. Simona Gurzu MD, PhD \\ Department of Pathology \\ University of Medicine \\ and Pharmacy \\ 38 Ghe Marinescu St \\ 540139 Tirgu-Mureş \\ Romania \\ Phone: +40745673550 \\ E-mail: \\ simonagurzu@yahoo.com
}


tion (EMR) is preferred for non-metastatic intramucosal differentiated carcinomas smaller than $2 \mathrm{~cm}$, with a protruded aspect at the endoscopic examination. If the tumor is ulcerated, EMR is recommended for differentiated carcinomas smaller than $1 \mathrm{~cm}$, whereas endoscopic submucosal dissection (ESD) is indicated for larger non-metastatic tumors with invasion limited to the submucosa $[4,5]$. The European criteria used for the endoscopic resection of EGC or surgery were outlined in the Paris endoscopic classification of superficial neoplastic gastrointestinal lesions published in 2003 [6]. However, although EMR and ESD are performed in some European medical institutes, open gastrectomy is mostly considered a standard therapy for any stage of GC, including EGCs [4, 7]. Long-term follow-up is necessary to examine the potential benefits of EMR/ESD vs. gastrectomy, as well as extension of lymph node dissection.

In this article, the prognostic value of some clinicopathologic factors and immunohistochemical (IHC) features are explored in patients with surgically removed EGCs diagnosed in three medical centers in Romania, Poland, and Hungary. Based on our own experience and literature data, an EGC-adapted staging system is proposed.

\section{Material and methods}

\section{Patients and tissue samples}

The surgically removed GC cases were retrospectively collected from three medical centers in Europe: the Clinical County Emergency Hospital of Tirgu-Mures, Romania and two Institutes of On-

Table I. Immunohistochemical antibodies used in the study

\begin{tabular}{|lcc|}
\hline Antibody (company) & Clone & Dilution \\
\hline $\begin{array}{l}\text { p53 (LabVision, } \\
\text { Fremont, CA, USA) }\end{array}$ & DO-7 & RTU \\
\hline Ki67 (LabVision) & SP6 & RTU \\
\hline $\begin{array}{l}\text { CD31/PECAM-1 } \\
\text { (LabVision) }\end{array}$ & JC/70A & $1: 25$ \\
\hline $\begin{array}{l}\text { CD105/endoglin } \\
\text { (Novocastra) }\end{array}$ & Mouse monoclonal & $1: 25$ \\
\hline VEGF-A (LabVision) & VG1 & $1: 50$ \\
\hline COX-2 (Novocastra) & Monoclonal & $1: 100$ \\
\hline $\begin{array}{l}\text { HER-2 (Dako } \\
\text { Glostrup, Denmark) }\end{array}$ & cerbB2-oncoprotein & $1: 1000$ \\
\hline MLH-1 (Novocastra) & ES05 & $1: 100$ \\
\hline MSH-2 (Novocastra) & $25 D 12$ & $1: 50$ \\
\hline E-cadherin (Dako) & NCH-38 & $1: 50$ \\
\hline Vimentin (Dako) & V9 & $1: 800$ \\
\hline RTU-ready to use. & & \\
\hline
\end{tabular}

cology - Warsaw, Poland and Budapest, Hungary. The IHC stains were performed on the paraffin blocks collected at the Department of Pathology of the University of Medicine and Pharmacy of Tirgu-Mures, Romania. The head of the three departments approved the processing of the cases, and the approval of the Ethical Committee of the University of Medicine and Pharmacy of Tirgu-Mures, Romania, was obtained.

The clinicopathological and IHC features of EGC were retrospectively evaluated in 29 EGCs selected from 338 GC (8.5\%) patients who underwent partial or total gastrectomy with lymph node dissection. This material included the 338 consecutive case series from the three medical centers, with a median follow-up time of 36 months (range: 2-61 months). From these cases, we selected all consecutive patients diagnosed postoperatively with EGC. All of the cases were surgical specimens; biopsy specimens were not taken into account. Patients who died in the first month after surgery were not included in this case series.

For tumor staging, the WHO/AJCC staging system (2010) was used, based on the invasion of the mucosa (pT1a) and submucosa (pT1b) $[2,3]$, and a sub-classification was done based on Japanese rules, with sub-categorizing in pT1a-m1 (invasion of upper third of mucosa), pT1a-m2 (middle third of mucosa), pT1a-m3 (lower third of mucosa), pT1b-sm1 (upper third of submucosa), pT1b-sm2 (middle third of submucosa), and pT1b-sm3 (lower third of submucosa) [4].

Although histopathological diagnoses were performed according to the WHO 2010 classification [2, 3], from the category of "poorly cohesive carcinoma", those with features of "signet ring cell carcinoma" were listed as a separate subtype. To certify the presence of mucinous carcinomas or a mucinous component, PAS/alcian blue staining was performed for all of the cases.

All of the metastases were biopsy proven. In lymph node negative cases, 5 sections were performed for any sentinel lymph node.

The IHC stains were performed using the antibodies mentioned in Table I and the Novolink Polymer Detection System (Novocastra, Newcastle Upon Tyne, UK) according to the instructions of the manufacturer. The developing was done with DAB (diaminobenzidine) solution (Novocastra). For the negative controls, incubation was done with the omission of specific antibodies. The IHC assessment was performed by two pathologists using the criteria described previously in the literature [8]. Microvessel density (MVD) was measured with CD31 and CD105, using the image analysis software ImageJ (NIH); the percentage of positive endothelial area versus the total area of the microscopic field was determined [8]. 


\section{Statistical analysis}

Statistical analysis was done with the GraphPad InStat 3 software and two-sided tests. A $p$-value $<0.05$ with $95 \%$ confidence interval was considered statistically significant. The patients' characteristics and IHC descriptions were presented using descriptive statistics. The results were presented using the mean value or were expressed as mean \pm standard deviation. The univariate analysis was performed with Pearson's or Student's t-test. For multiple associations the Fisher exact test was used, and the correlations between two variables were performed using the Mann-Whitney test. Multivariate logistic regression analysis and the log-rank test were used to evaluate the independent prognostic factors. Tumor-related death was used to explore the overall survival rate.

\section{Results}

\section{Clinicopathological characteristics}

As mentioned, the 29 patients with EGCs were selected from 338 GC (8.5\%) patients diagnosed in the three European institutes. In Romania, they represented $5.61 \%$ of all GCS (11 patients with EGCs from a total of 196 GCs), whereas in Poland and Hungary the percentage was higher, constituting $12.37 \%$ (12 EGCs from a total of 97 GCs) and $13.33 \%$ ( 6 EGCs from a total of 45 GCs) respectively.

Examining the 29 patients with EGCs, a male predominance was noted, with a male/female ratio of $1.6: 1$ (18 males and 11 females). Independently from the patient's geographic origin, males were diagnosed at a significantly younger age (mean age: $58.61 \pm 10.05$ years; range: $36-75$ years) than the females (mean age: $67.18 \pm 11.71$ years; range: $42-80$ years) $(p=0.04)$ (Table II).

Most of the cases $(n=18 ; 62.07 \%)$ were localized in the distal stomach, the proximal stomach being mostly predominant in patients from Poland. The mean tumor size was $4.26 \pm 2.45 \mathrm{~cm}$ (range: $0.5-9 \mathrm{~cm}$ ).

The signet ring cell subtype was seen in 7 (24.14\%) cases, while poorly cohesive non-signet ring cell carcinoma was diagnosed in only 2 patients from Poland, 1 in the proximal stomach and 1 in the antrum (cases no. 13 and 19). Comparing the 7 signet ring cell carcinomas with the intestinal-type adenocarcinomas, no significant differences were seen regarding the mean age (58.85 \pm 8.97 years (range: $45-72$ years) vs. 63.65 \pm 11.61 years (range: $36-80$ years); $p=0.33$ ) or other clinicopathologic parameters. Only 6 of the $29(20.69 \%)$ cases were limited to mucosa; 2 of them (cases no. 10 and 17), located in the deep mucosa (pT1a-m3), were metastatic (Table II).
Seven out of the 29 (24.14\%) patients presented angio-lymphatic invasion, 6 of them also having metastases in the regional lymph nodes. Regarding the distant metastases, 2 patients (cases no. 9 and 10) had liver metastases, whereas case no. 8 had a skip metastasis in the non-regional lymph node located along the middle colic artery (station 15) [9]. The first patient with multicentric liver metastasis (case no. 9) had a multifocal adenocarcinoma with minute submucosal invasion (pT1b-sm1). He was postoperatively treated with chemotherapeutics and hepatic chemoembolization and died at 14 months of follow-up. The second patient (case no. 10) presented a multifocal signet ring cell carcinoma limited to the mucosa (pT1a-m3), without invasion of the muscularis mucosae, but extensive intramural carcinomatosis of the lymph vessels located in the submucosa, muscularis, and serosa was noted (Figure 1). This aspect was associated with metastases in all of the 48 regional D2-lymph nodes and 1 liver metastasis that were surgically removed. With chemotherapy, the patient is still alive at the $6^{\text {th }}$ month of follow-up. The third patient (case no. 8) presented a pT1b-sm 2 staged adenocarcinoma and was postoperatively treated with chemotherapy. She is alive at the $10^{\text {th }}$ month of follow-up.

\section{Immunohistochemistry}

In all of the 29 cases, investigation of the possible prognostic value of the positive E-cadherin, HER-2, p53, Ki67, VEGF-A (vascular endothelial growth factor), and COX-2 was performed. The mean MVD value was $3.11 \pm 1.23$ for CD31 and $2.61 \pm 1.56$ for CD105, without a correlation between the two endothelial markers $(p=0.18)$. VEGF-A positivity was seen in 15 (51.72\%) of the 29 cases but was not correlated with MVD for either CD31 $(p=0.26)$ or CD105 ( $p=0.83)$. COX-2 positivity was observed in 14 (48.27\%) cases, without a correlation with VEGF positivity. The MVD counted with CD31 was significantly higher in COX-2 positive vs. COX-2 negative cases (4.15 \pm 0.24 vs. $2.14 \pm 0.11 ; p<0.0001)$. A positive correlation was also found between COX-2 and CD105 (3.46 \pm 0.47 vs. $1.82 \pm 0.19 ; p=0.03$ ).

HER-2 positivity $(+++)$ was observed in only $6(20.69 \%)$ of the 29 cases: 1 mucinous adenocarcinoma (case no. 14), 2 signet ring cell carcinomas (cases no. 1 and 7), and 3 moderately differentiated adenocarcinomas (cases no. 2, 6, and 11). None of the 6 cases presented metastases. Meanwhile, all of them expressed VEGF and COX-2 and presented p53 and Ki67 positivity. MVD was significant higher in the HER-2 positive compared with HER-2 negative cases (Figure 2), for both CD31 (4.36 \pm 0.4 vs. $2.78 \pm 0.22 ; p=0.03)$ and CD105 (4.04 \pm 0.54 vs. $2.24 \pm 0.29 ; p=0.0007)$. 


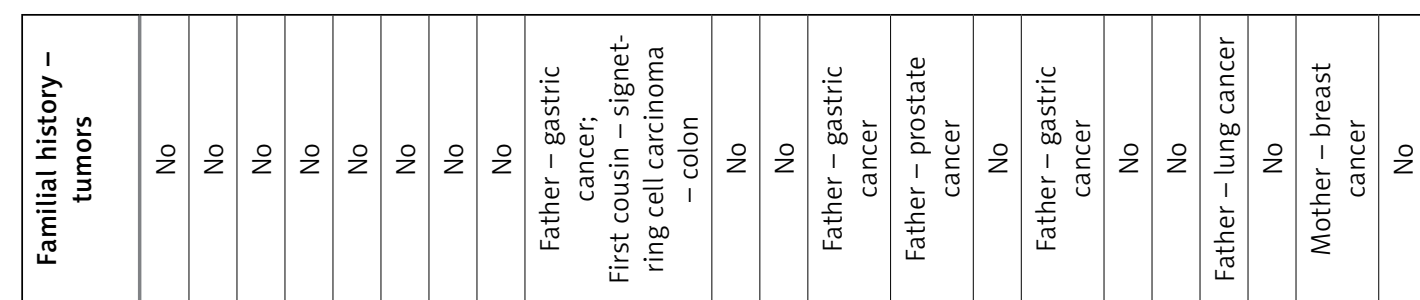

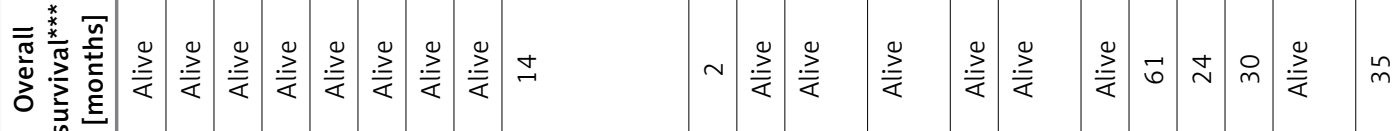

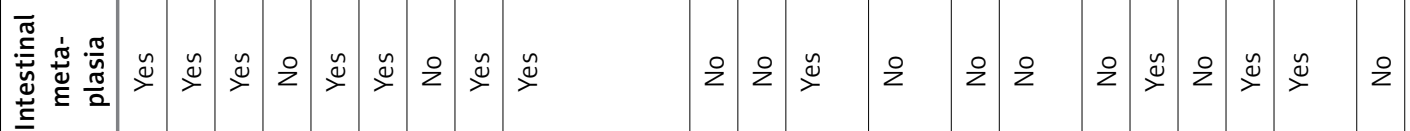

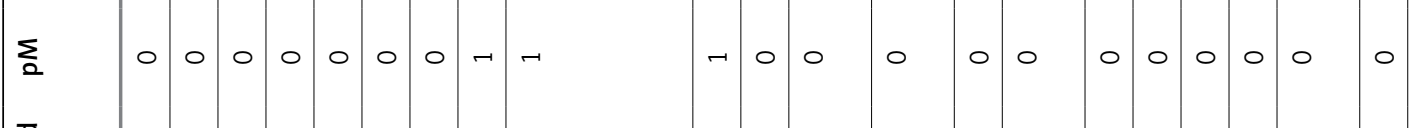

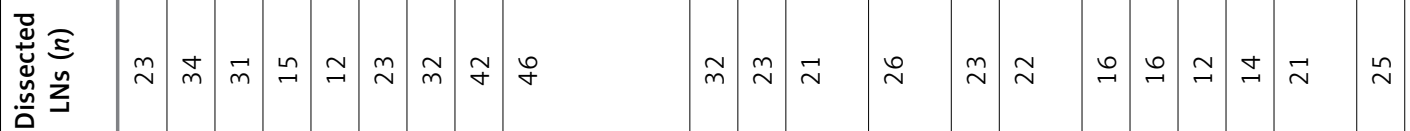

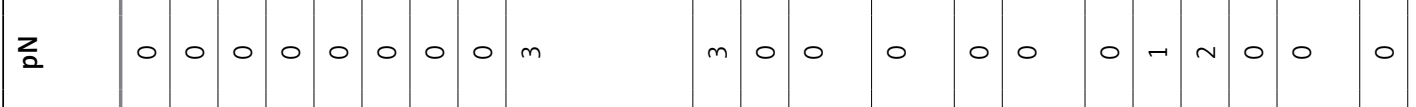

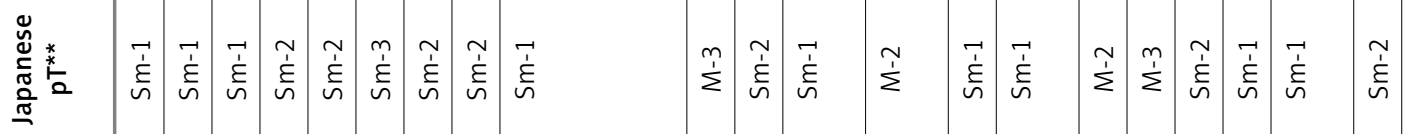

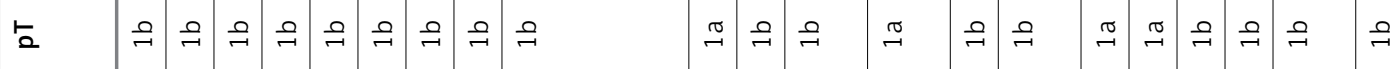

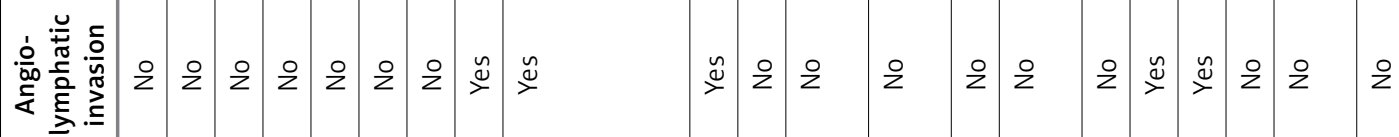

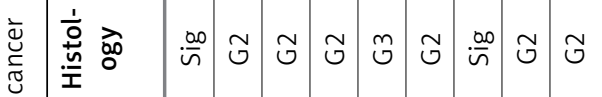

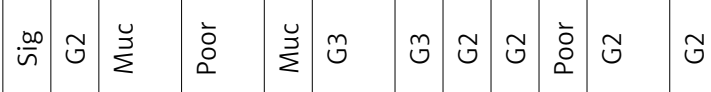

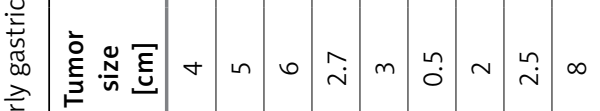

$\stackrel{*}{\circ} \quad \equiv \equiv-\equiv-$

$\rho$ 满

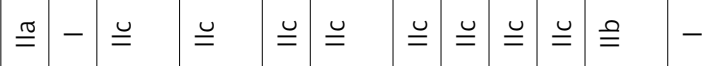

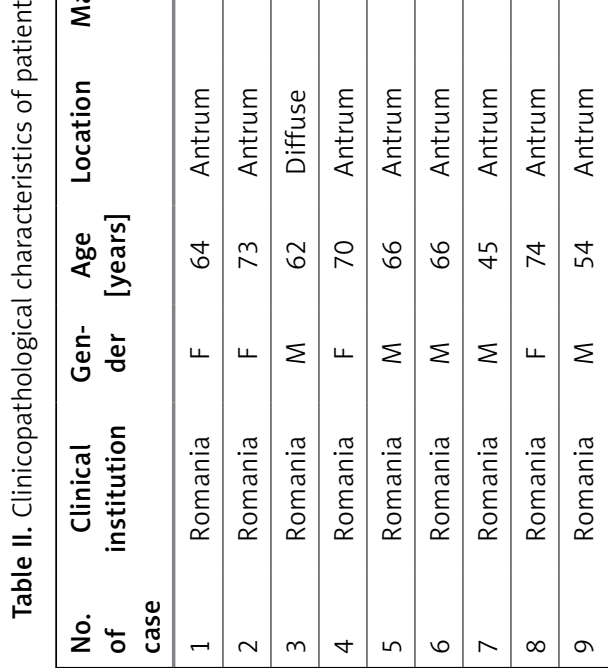

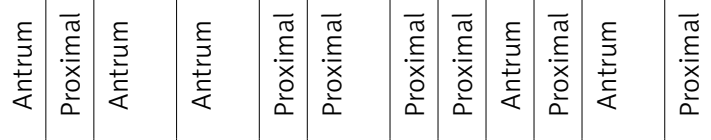

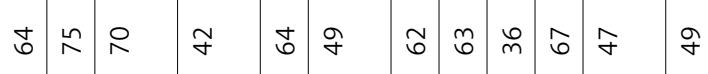

$\Sigma \Sigma \Sigma \quad \sqcup \quad \Sigma \Sigma \quad \sqcup \Sigma \Sigma \Sigma \Sigma \Sigma$

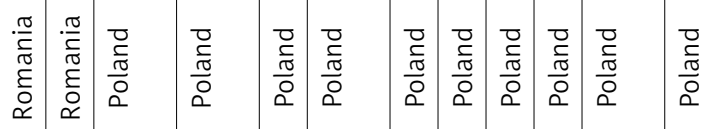

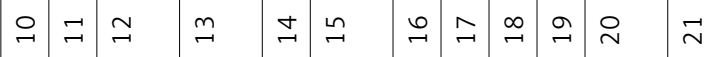




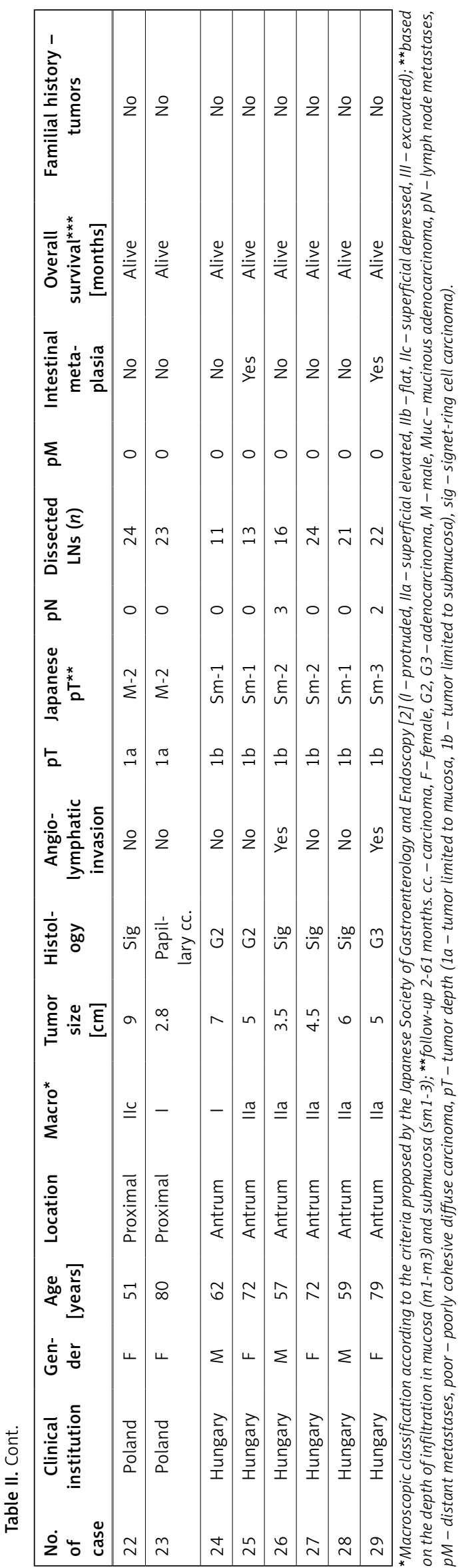

Positivity for p53 and Ki67 was observed in 16 (55.17\%) and 14 (48.27\%) cases respectively. Two of the 4 tumors of the gastroesophageal junction (GEJ) expressed COX-2 and were Ki67/p53 negative (cases no. 16 and 23). Both of the patients are still alive after 22 months of follow-up. The other two COX-2 negative/p53 negative/Ki67 positive EGCs of the GEJ patients presented associated intestinal metaplasia (cases no. 17 and 19) and died at 61 and 30 months respectively. None of the 29 cases expressed vimentin, and all of them showed positivity for both markers of microsatellite status, MLH-1 and MSH-2. E-cadherin was totally lost in case no. 7 (a 45-year-old man with a pT1b-sm2 signet ring cell carcinoma without a familial history of any neoplasia), the other cases being positive (Figure 1).

\section{Overall survival rate}

None of the 29 patients presented local recurrence. Six (20.69\%) out of the 29 patients died at a median time of 27 months after surgery (ranging between 2 and 61 months) (Table II). All of these tumors were negative for p53 and HER-2.

Only tumor localization was proved to have independent prognostic value $\left(p=0.03, \chi^{2}=6.44\right.$, degrees of freedom (DF) $=2$ ) among all of the analyzed parameters. The longest survival was noted for antral EGC patients, while the proximal localization proved to be associated with the lowest survival rate, independently from other parameters. Slightly longer survival was also found in females, regardless of age, compared with males ( $p=0.04, \chi^{2}=4.30$, $\mathrm{DF}=1$ ) (Figure 3 ). When the pT1a cases were analyzed, the subdivision into $\mathrm{m} 1, \mathrm{~m} 2$ and $\mathrm{m} 3$ did not prove to have prognostic value, and nor did pT1b subdivision into sm $1, \mathrm{sm} 2$ and sm3. Moreover, it was difficult to separate $\mathrm{m} 2$ from $\mathrm{m} 3$ and $\mathrm{sm} 2$ from $\mathrm{sm} 3$.

There was no correlation between the overall survival rate and any of the following immunohistochemical markers: Ki-67 $\left(p=0.39, \chi^{2}=0.72\right.$, $\mathrm{DF}=1)$, p53 $\left(p=0.26, \chi^{2}=1.22\right.$, DF $\left.=1\right)$, HER-2 $\left(p=0.23, \chi^{2}=1.43\right.$, DF $\left.=1\right)$, COX-2 $(p=0.66$, $\left.\chi^{2}=0.18, \mathrm{DF}=1\right)$, and $\operatorname{VEGF}\left(p=0.35, \chi^{2}=0.83\right.$, $\mathrm{DF}=1$ ) (Figure 3).

\section{Discussion}

The lack of GC screening programs leads to the diagnosis of less than $15 \%$ of tumors in their early stages in Europe [7], including Poland and Hungary $(12 \%$ and $13 \%$ of all GCS) and even a lower percentage in Romania (5.6\%), as demonstrated in the present report. In contrast, the proportion of EGC is much higher, up to $40-50 \%$ of all GCs in Japan and South Korea [4, 5, 10, 11].

Although about $24 \%$ of EGCs have a signetring cell subtype in European patients (24.14\% 

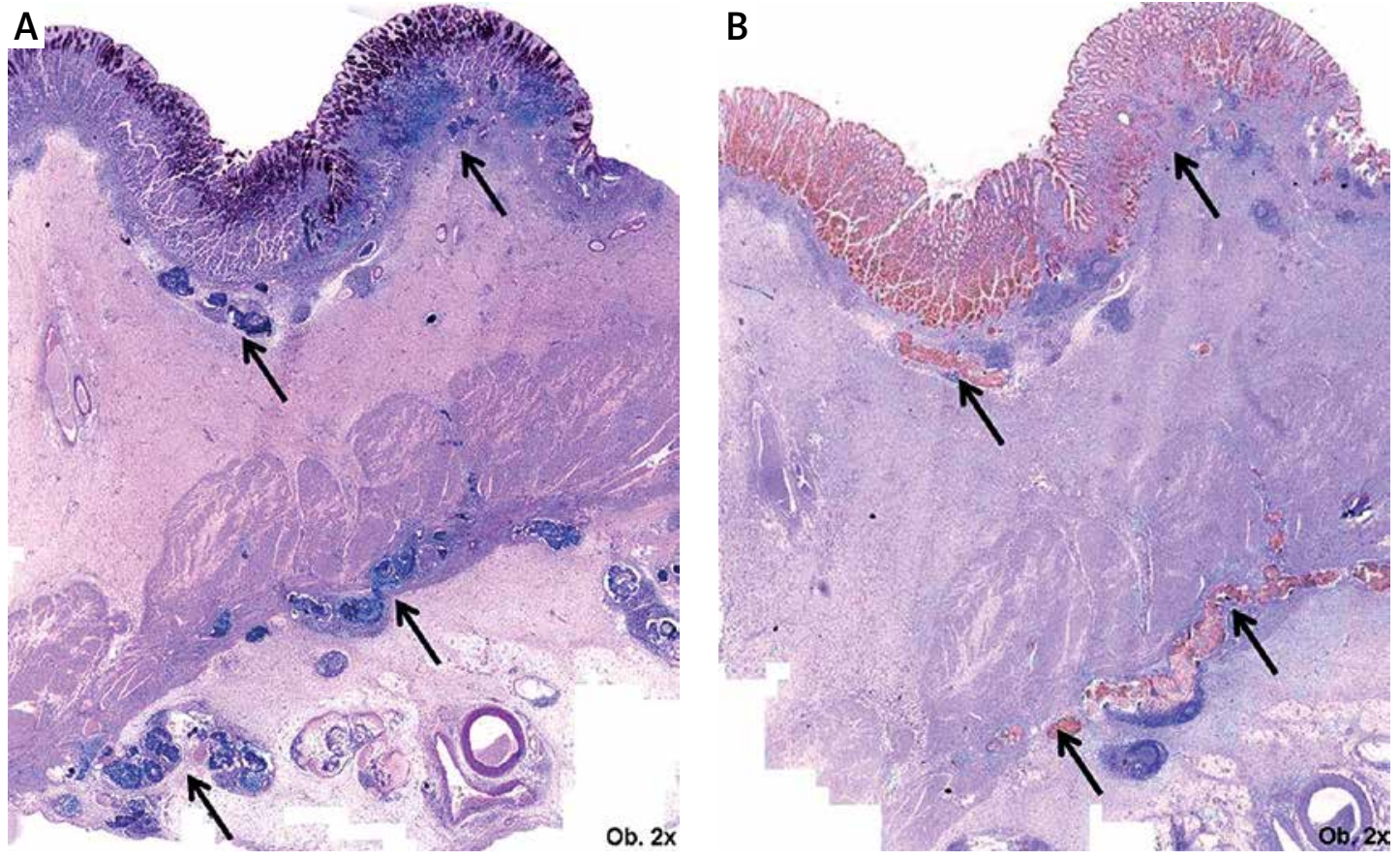

Figure 1. In a gastric cancer with direct invasion limited to the deep mucosa (pT1a-m3), extensive intramural carcinomatosis of the lymph vessels can be noted in blue in the submucosa muscularis propria, and subserosa (case 10), with PAS/alcian stain (A) and in brown with E-cadherin (B)
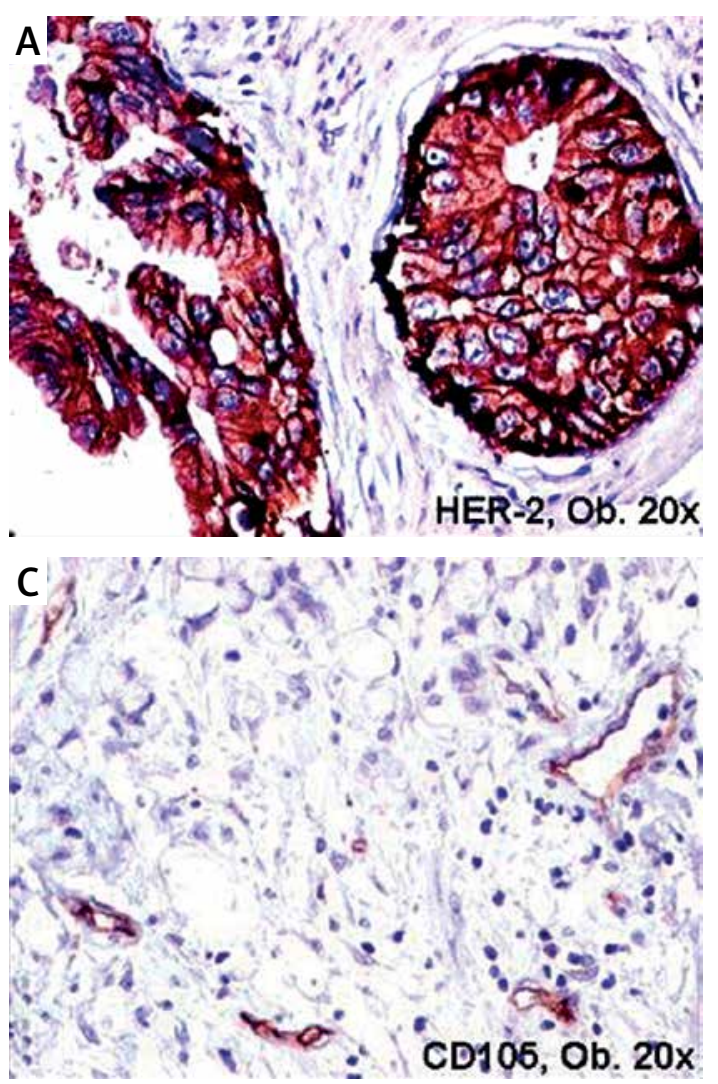

$(n=7)$ in our material), the histological variant seems to not influence the 3-year survival rate in the pT1 stage [12]. In larger cohorts but not in our group of patients, it was proved that the signet ring cell carcinomas, compared to the

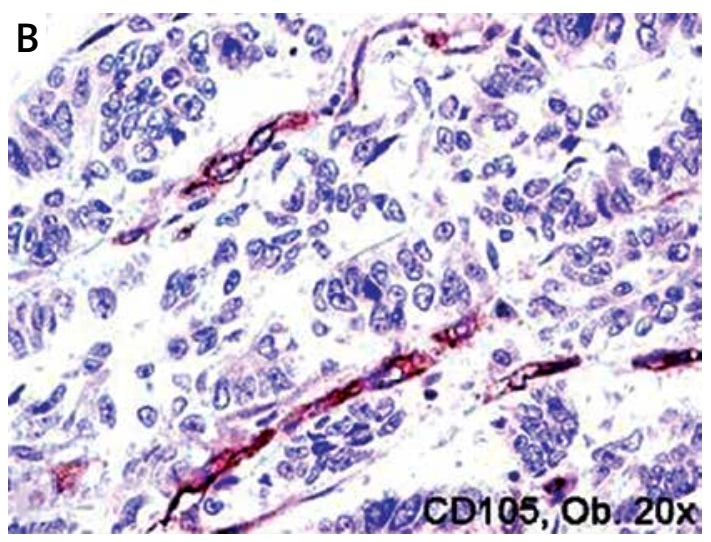

Figure 2. In an intestinal-type carcinoma that showed HER-2 positivity (A) the microvessel density is relatively high (B), compared with a HER-2 negative diffuse gastric cancer (C)

non-signet ring cell subtypes, occur in younger patients, and the submucosa is more frequently involved. There is a disagreement concerning the number of invaded lymph nodes and the relapse rate, if they are independent from the histologic 

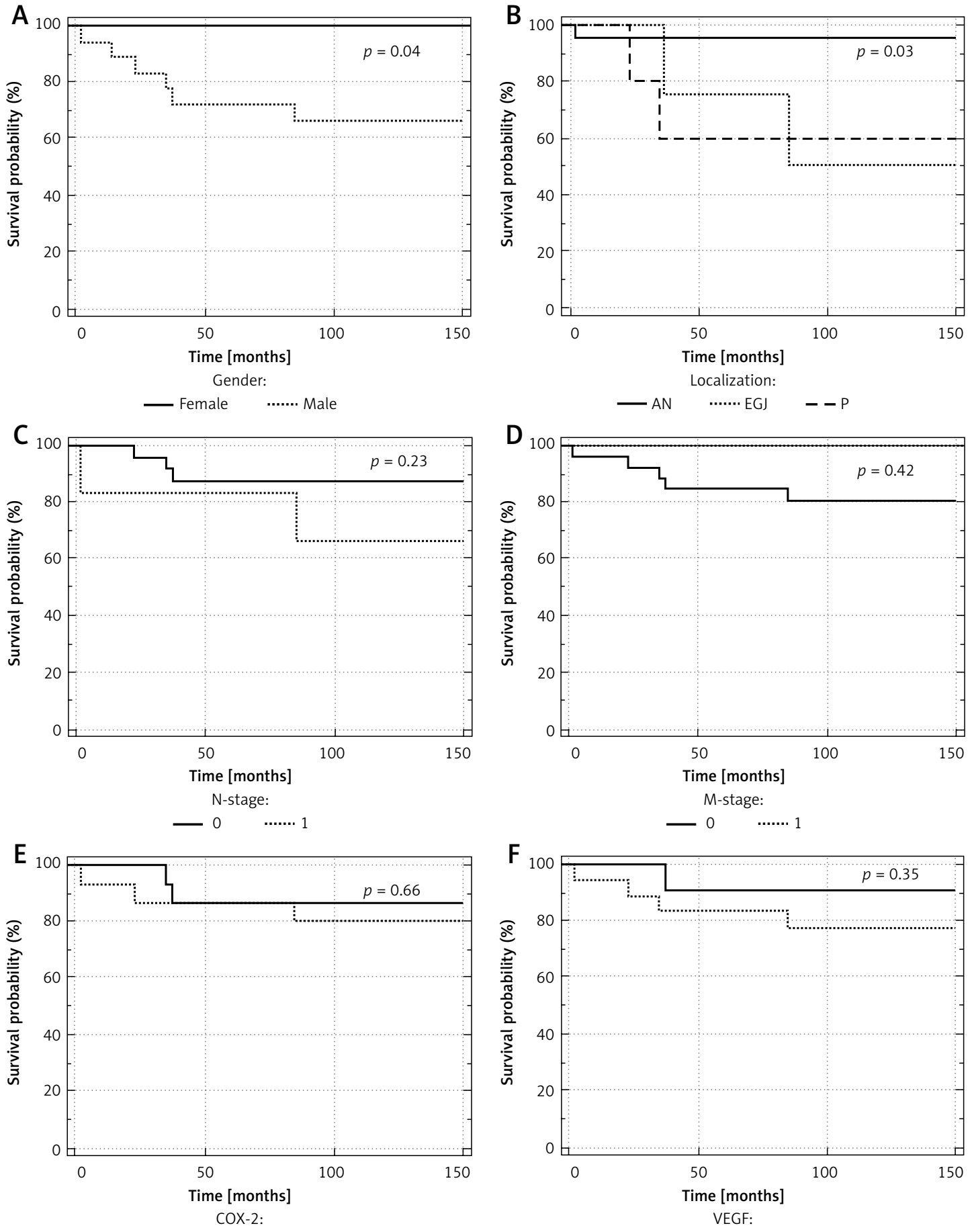

- 1 -...... 2

- $1 \quad \cdots \cdots \cdot 2$

Figure 3. In patients with early gastric cancer, the overall survival rate is directly correlated with patient's gender (A) and tumor localization (B) but does not depend on the lymph node status (C), distant metastases (D), or any of the following immunohistochemical markers: COX-2 (E), VEGF-A (F)

AN - antrum, EGJ - esophagogastric junction, $N$ - lymph node status, $M$ - distant metastases, $P$ - proximal, 1 - positive immunostain, 2 - negative stain.

subtype [12] or higher in signet ring cell carcinomas [5]. In Japanese patients with EGC, about $15 \%$ of the cases were proved to be signet ring cell carcinomas, and their prognosis was significantly worse compared to the non-signet ring cell variants [10].
Despite the several studied parameters, age and PTNM stage remain the most important independent factors for predicting the survival of patients with EGC $[10,13-16]$. In our material, the lymph node metastatic rate was $20.69 \%$. Only 2 of the 6 metastatic cases were diagnosed in the 


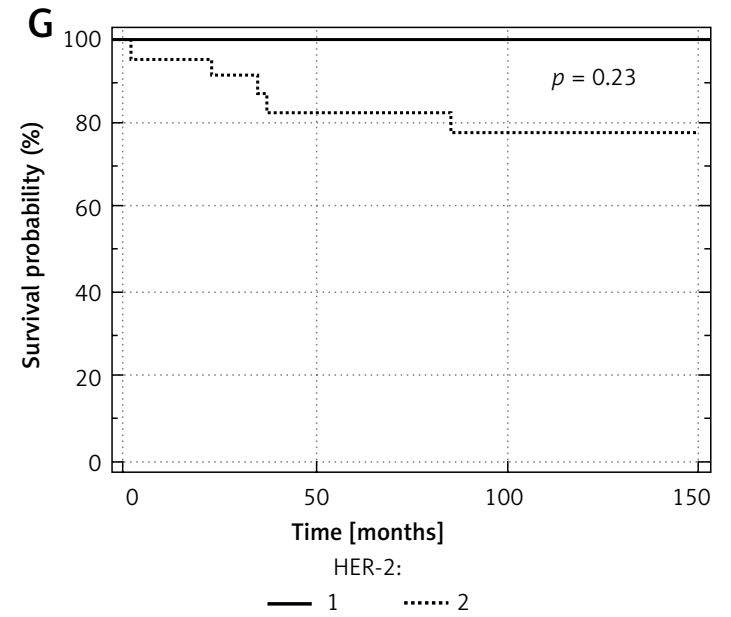

Figure 3. Cont. HER-2 (G), and Ki67 (H)

pT1a-m3 stage; the others were T1b-staged. This rate was higher than most of the previously published studies, which revealed lymph node positivity in less than $10 \%$ of the cases; $3-4 \%$ for pT1a and $17-20 \%$ for pT $1 \mathrm{~b}$ carcinomas $[4,5,13]$. The independent predictive risk factors for lymph node involvement are the depth of infiltration (pT1a-m3 and $\mathrm{pT} 1 \mathrm{~b}$ stages), presence of angiolymphatic invasion, ulceration, tumor size larger than 2-3.5 $\mathrm{cm}$, undifferentiated histology, and expression of epidermal growth factor receptor (EGFR) [2, 5, 1316]. However, grouping of the cases based on one third of the mucosa or of the submucosa is very difficult. Moreover, because $\mathrm{m} 3$ and sm3 were mostly shown to have prognostic value, compared with $\mathrm{m} 1 / 2$ and $\mathrm{sm} 1 / 2$ respectively, we consider that grouping in two categories based on the upper and lower part of the mucosa and submucosa, respectively, would be more practical for daily activity (Table III).

In our material, 7 of the 29 patients presented angiolymphatic invasion. From them, extended intramural carcinomatosis of the lymph vessels was seen in 1 patient who died 2 months after surgery. Because angio-lymphatic invasion is a significant prognostic parameter for patients with EGC [2, 14-19] it should be separately described in the pathologic reports of EGC (Table III).

The adequate postoperative therapy of patients with EGC may also be influenced by a staging system specifically adapted for EGC. At the moment, according to the National Comprehensive Cancer Network (NCCN) guidelines version 3.2015, only postoperative follow-up is recommended for patients diagnosed with pT1NO stage gastric cancer who underwent surgery, independently from other parameters, except the resection margins [20]. According to the European guidelines developed by the European Societies of Medical Oncology (ESMO), Surgical Oncology (ESSO), and Radiotherapy and Oncology (ESTRO), endoscopic resection

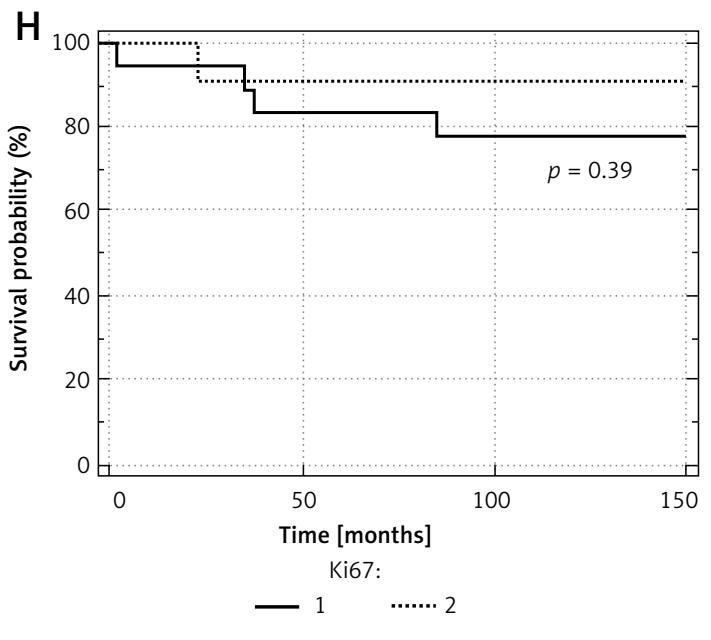

is recommended for pT1N0 tumors smaller than $2 \mathrm{~cm}$ and preoperative chemotherapy is advised for the other cases. However, angiolymphatic invasion is not taken into account for the postoperative evaluation and surveillance, and the latest update was done in 2014 [21].

Another parameter that should be attentively examined in patients with EGC is the tumor multifocality or multicentricity. This characteristic was especially observed in hereditary diffuse gastric cancers [22], but acquired diffuse cancers can also have a multicentric aspect, being known as "carcinomas with intraepithelial pagetoid onset" [19]. Because the intramucosal foci are difficult to detect endoscopically [19, 22], description of multifocality in the histopathological report is a valuable parameter for endoscopic follow-up of patients who have undergone endoscopic resection or subtotal gastrectomy.

Regarding the immunoprofile, loss of E-cadherin, considered as an indicator of tumor progression [23], was seen in only one signet ring cell EGC at an early onset. The angiogenic activity was proved to be intense in half of the cases diagnosed in early stages. All of the COX-2 positive cases displayed high microvessel density. This aspect could support the possible prophylactic role of standard doses of anti-COX-2 substances, including aspirin, in reducing the overall cancer incidence in at least 3-year users. Long-term use is indicated to be prescribed after Helicobacter pylori eradication [24]. Moreover, in our material, 2 of the 4 tumors from the proximal stomach showed COX-2 but not HER-2 positivity (cases no. 16 and 19); neither of them was associated with intestinal metaplasia. This aspect could also sustain the role of anti-COX-2 substances in preventing gastric and/or esophageal carcinogenesis but not association of nonsteroidal anti-inflammatory drugs with a decreased risk of Barrett esophagus [24, 25]. Long-term use of anti-COX-2 substances could have a protective 
Table III. Proposal for an early gastric cancer adapted pTNM staging system based on the currently used WHO/ AJCC systems [2, 3], our experience, and criteria from Japanese and European literature [1, 4-26] (the proposed modified criteria are emphasized with bold + italic; these criteria are not available for advanced tumors, currently classified as PT2-T4)

\begin{tabular}{|c|c|}
\hline Parameter & Significance \\
\hline Tis & Carcinoma in situ: intraepithelial tumor without invasion of the lamina propria \\
\hline T1a & Tumor invades lamina propria or muscularis mucosae $(\mathrm{m})$ \\
\hline $\begin{array}{l}T 1 a-m 1 \\
T 1 a-m 2\end{array}$ & $\begin{array}{l}\text { - Invasion of the upper half of the mucosa } \\
\text { - Invasion of the lower half of the mucosa }\end{array}$ \\
\hline T1b & Tumor invades submucosa (sm) \\
\hline $\begin{array}{l}T 1 b-s m 1 \\
T 1 b-s m 2\end{array}$ & $\begin{array}{l}\text { - Invasion of the upper half of the submucosa } \\
\text { - Invasion of the lower half of the submucosa }\end{array}$ \\
\hline NO & No regional lymph node metastasis \\
\hline$N 1^{*}$ & Metastasis in $1-2$ regional lymph nodes \\
\hline $\begin{array}{l}N 1 a \\
N 1 b \\
N 1 c\end{array}$ & $\begin{array}{l}\text { - Metastasis in 1-2 regional lymph nodes } \\
\text { - Micrometastasis in 1-2 regional lymph nodes } \\
\text { - Isolated tumor cells in 1-2 regional lymph nodes }\end{array}$ \\
\hline N2 & Metastasis in 3-6 regional lymph nodes \\
\hline N3 & Metastasis in 7 or more regional lymph nodes \\
\hline $\begin{array}{l}\text { N3a } \\
\text { N3b }\end{array}$ & $\begin{array}{l}\text { - Metastasis in } 7-15 \text { regional lymph nodes } \\
\text { - Metastasis in } 16 \text { or more regional lymph nodes }\end{array}$ \\
\hline$L C-N O-N 3^{* *}$ & $\begin{array}{c}\text { Intramural carcinomatosis of the lymph vessels from the submucosa and/or muscularis propria, } \\
\text { regardless of the status of the lymph nodes }\end{array}$ \\
\hline MO & No distant metastases \\
\hline M1 & Distant metastases \\
\hline $\begin{array}{l}\text { M1a } \\
M 1 b \\
M 1 c\end{array}$ & $\begin{array}{c}\text { - Metastasis in one or more distant lymph nodes or skip metastasis } \\
- \text { metastasis in one organ } \\
- \text { metastasis in multiple organs }{ }^{* * * *}\end{array}$ \\
\hline
\end{tabular}

*Micrometastasis refers to metastasis greater than $0.2 \mathrm{~mm}$ but smaller than $2 \mathrm{~mm}$; ${ }^{* *}$ it is only reported for tumors with invasion limited to the mucosa, without direct invasion of the submucosa (pT1a); ${ }^{* * i}$ in case of bone metastases, solitary metastasis; ${ }^{* * *}$ in case of bone metastases, multicentric metastasis. $L C$ - lymphatic carcinomatosis, $m$ - mucosa, $M$ - distant metastasis, $N$ - regional lymph nodes, sm - submucosa, $T$ - depth of infiltration of the primary tumor.

role for the gastric mucosa. On the other hand, non-Barrett related advanced tumors of the gastro-esophageal junction seem to not respond to trastuzumab. It is also worth noting that HER-2 positivity, shown by more than $25 \%$ of the EGC cases $[5,8]$, as well as the p53 index did not prove to indicate higher aggressiveness in EGC, as described in the advanced stages [5].

The clinical importance of angiogenesis is also sustained by magnifying endoscopy with narrow-band imaging used for diagnosis of EGC. This method proved that more than $90 \%$ of patients with EGC present irregular microvascular patterns referring to dilation or variation in diameter or shape and about one third of them show microvessel tortuosity [18]. Examining microvessel density and the morphological aspect could provide diagnostic and prognostic parameters for patients with EGC. Moreover, the highly angiogenic potential should be used as a therapeutic indicator, VEGF having pro-angiogenic and pro-inflam- matory properties combined with its capacity for enhancing free radical generation [26].

Based on our results and literature data, we propose adapting the existent WHO/AJCC pTNM staging system used for GC $[2,3]$ to be specifically used for EGC (Table III). This is a preliminary proposal, based on the findings of this study but also the daily experience of the five Professors in Pathology from Romania, Poland, Hungary, and Japan, which should be amended in the future.

The main weak point of the study is the small number of analyzed cases, which does not allow a detailed statistical analysis. However, the obtained results highlight the aggressiveness of EGC, which can be associated not only with the multifocal aspect, aberrant lymphatic flow, and skip metastasis but also with localization in the proximal stomach, independently from the microsatellite status, HER-2, E-cadherin, p53, or Ki67 expression. The angiogenic pattern could be used as a therapeutic target of EGC. 


\section{Acknowledgments}

This work was partially supported by the team research project frame UMFTGM-PO-CC02-F01-19/2014 funded by the University of Medicine and Pharmacy of Tirgu-Mures, Romania and Smoking Research Foundation (SRF), MEXT (S-001), and A-MED, from Japan. We acknowledge Prof. Pavai Zoltan, Ms. Marika Jung, and Ms. Emese Nagy for technical assistance. The English language manuscript was polished by SPI Global Professional Editing Service.

\section{Conflict of interest}

The authors declare no conflict of interest.

\section{References}

1. Begenik H, Aslan M, Dulger AC, et al. Serum leptin levels in gastric cancer patients and the relationship with insulin resistance. Arch Med Sci 2015; 11: 346-52.

2. Bosman FT, Carneiro F, Hruban RH, Theise ND (eds). Tumors of the stomach. In: WHO Classification of Tumours of the Digestive System. World Health Organization Classification of Tumours. Lyon, France: IARC, 2010; 45-80.

3. American Joint Committee on Cancer. Stomach. In: AJCC Cancer Staging Handbook. $7^{\text {th }}$ ed. Edge SB, Byrd DR Compton CC, Fritz AG, Greene FL, Trotti A (eds.). Springer, New York 2010; 145-52.

4. Yada T, Yokoi C, Uemura N. The current state of diagnosis and treatment for early gastric cancer. Diagn Ther Endosc 2013; 2013: 241320.

5. Jin EH, Lee DH, Jung SA, et al. Clinicopathologic factors and molecular markers related to lymph node metastasis in early gastric cancer. World J Gastroenterol 2015; 21: 571-7.

6. Participants in the Paris Workshop: The Paris endoscopic classification of superficial neoplastic lesions: esophagus, stomach, and colon: November 30 to December 1, 2002. Gastrointest Endosc 2003; 58 (Suppl.): 3-43.

7. Koessler T, Roth A, Cacheux W. Early gastric cancer: epidemiology, diagnostic and management. Rev Med Suisse 2014; 10: 1118-22.

8. Gurzu S, Kadar Z, Sugimura H, et al. Maspin-related orchestration of aggressiveness of gastric cancer. Appl Immunohistochem Mol Morphol 2015; DOI: 10.1097/ PAl.0000000000000189.

9. Bara T Jr, Gurzu S, Jung I, Kadar Z, Sugimura H, Bara T. Single skip metastasis in sentinel lymph node, in an early gastric cancer. World J Gastroenterol 2015; 21: 9803-9807.

10. Kwon KJ, Shim KN, Song EM, et al. Clinicopathological characteristics and prognosis of signet ring cell carcinoma of the stomach. Gastric Cancer 2014; 17: 43-53.

11. Association TICotKGC. 2004 Nationwide gastric cancer report in Korea. J Korean Gastric Cancer Assoc 2007; 7: 47-54.

12. Triboulet JP, Gronnier C, Messager M, Piessen G, Mariette C, FREGAT Working Group. Does early signet ring cell gastric carcinoma carry a worse prognosis? Bull Acad Natl Med 2013; 197: 443-55.

13. Okabayashi T, Kobayashi M, Nishimori I, et al. Clinicopathological features and medical management of early gastric cancer. Am J Surg 2008; 195: 229-32.
14. Godota T, Yanagisawa A, Sasako M, et al. Incidence of lymph node metastasis from early gastric cancer: estimation with a large number of cases at two large centers. Gastric Cancer 2000; 3: 219-25.

15. Hirota T, Ming SC. Early gastric carcinoma. In: Pathology of the gastrointestinal tract. Ming SC, Goldman H (eds). Williams \& Wilkins, 1998; 570-83.

16. Li H, Lu P, Lu Y, Xu HM, Wang SB, Chen JQ. Predictive factors for lymph node metastasis in poorly differentiated early gastric cancer and their impact on the surgical strategy. World J Gastroenterol 2008; 14: 4222-6.

17. Gurzu S, Jung I, Kadar Z. Aberrant metastatic behavior and particular features of early gastric cancer. APMIS 2015; 123: 999-1006.

18. Kanesaka T, Uedo N, Yao K, et al. A significant feature of microvessels in magnifying narrow-band imaging for diagnosis of early gastric cancer. Endosc Int Open 2015; 3: E590-6.

19. Gurzu S, Sugimura H, Orlowska J, Szentirmay Z, Jung I. New insights in histogenetic pathways of gastric cancer. Medicine (Baltimore) 2015; 94: e1810.

20. http://www.nccn.org/framework. Accessed at January first, 2016.

21. Waddell T, Verheij M, Allum W, Cunningham D, Cervantes A, Arnold D, European Society for Medical Oncology (ESMO), European Society of Surgical Oncology (ESSO), European Society of Radiotherapy and Oncology (ESTRO). Gastric cancer: ESMO-ESSO-ESTRO clinical practice guidelines for diagnosis, treatment and follow-up. Ann Oncol 2014; 40: 584-91.

22. Gurzu S, Jung I, Orlowska J, et al. Hereditary diffuse gastric cancer - an overview. Pathol Res Pract 2015; 211: 629-32.

23. Yi Kim D, Kyoon Joo J, Kyu Park Y, et al. E-cadherin expression in early gastric carcinoma and correlation with lymph node metastasis. J Surg Oncol 2007; 96: 429-35.

24. Cuzick J, Thorat MA, Bosetti C, et al. Estimates of benefits and harms of prophylactic use of aspirin in the general population. Ann Oncol 2015; 26: 47-57.

25. Khalaf N, Nguyen T, Ramsey D, El-Serag HB. Nonsteroidal anti-inflammatory drugs and the risk of Barrett's esophagus. Clin Gastroenterol Hepatol 2014; 12: 1832-9.

26. Yu H, Liu Y, Pan W, Shen S, Das UN. Polyunsaturated fatty acids augment tumoricidal action of 5 -fluorouracil on gastric cancer cells by their action on vascular endothelial growth factor, tumor necrosis factor-alpha and lipid metabolism related factors. Arch Med Sci 2015; 11: 282-91. 